\title{
Exploring broad consent in the context of the 100,000 Genomes Project: a mixed methods study
}

\author{
Lisa M. Ballard $\mathbb{1}^{1} \cdot$ Rachel H. Horton ${ }^{1} \cdot$ Sandi Dheensa ${ }^{2} \cdot$ Angela Fenwick $^{1} \cdot$ Anneke M. Lucassen $\mathbb{I}^{1}$
}

Received: 19 August 2019 / Revised: 6 December 2019 / Accepted: 17 December 2019 / Published online: 9 January 2020

(c) The Author(s), under exclusive licence to European Society of Human Genetics 2020

\begin{abstract}
The 100,000 Genomes Project (100kGP)—a hybrid clinical-research initiative-was set up to analyse whole-genome sequences (WGS) from patients living with a rare disease or cancer. The project positioned participant consent as being of central importance, but consent in the context of genomic testing raises challenging issues. In this mixed method study, we surveyed 1337 100kGP participants regarding their experiences of taking part in the project and conducted in-depth interviews with 24 survey respondents to explore these findings further. Survey responses were analysed using descriptive statistics and interview data were analysed thematically. The consent approach of the 100kGP resulted in a proportion of our study's participants not understanding the complexities of the project and what types of results they might receive; for example, $20 \%$ of participants who we surveyed from the cancer arm did not recall what decisions they had made regarding additional findings. It is not surprising that a project such as this, with such diverse aims and participant groups, would throw up at least some challenges. However, participants reported being satisfied with their experience of the project to date. Our study highlights that in the context of consent for more complex endeavours, such as the 100kGP, it is important to assess (and document) an agreement to take part, but complicated decisions about what and when to communicate may need revisiting over time in response to changing contexts. We discuss the implications of our findings with reference to participants of the 100kGP and the newly formed NHS Genomic Medicine Service.
\end{abstract}

\section{Introduction}

The 100,000 Genomes Project $(100 \mathrm{kGP})^{1}$ was a hybrid clinical-research initiative set up to sequence whole genomes from National Health Service (NHS) patients initially in England, but later extended to include Wales, Scotland and Northern Ireland. The project aimed to find molecular

Supplementary information The online version of this article (https:// doi.org/10.1038/s41431-019-0570-7) contains supplementary material, which is available to authorized users.

Lisa M. Ballard

1.ballard@soton.ac.uk

1 Clinical Ethics and Law at Southampton (CELS), Faculty of Medicine, University of Southampton, Southampton, UK

2 Centre for Academic Primary Care, Population Health Sciences, Bristol Medical School, University of Bristol, Bristol, UK

\footnotetext{
${ }^{1}$ For more details regarding the $100 \mathrm{kGP}$ please visit www. genomicsengland.co.uk/about-genomics-england/the-100000genomes-project/.
}

genetic diagnoses for people affected by rare conditions, as well as to improve treatment and outcomes for people with cancer. Identifying the genetic cause of a suspected rare disease, or improved treatment for someone with cancer were the 'main findings' to be provided to participants. Participants could also opt to receive 'additional findings' (AFs) and carrier testing, the results of which are still to be released. AFs are selected genetic risk factors that predispose for serious conditions, for which screening and/or treatment are usually available. The list of AFs looked for is still subject to change, as evidence evolves, but currently includes various cancer predisposition syndromes and familial hypercholesterolaemia [1]. The NHS Genomic Medicine Service has been set up in England with similar aims and infrastructure to the 100kGP, though AFs will not initially be included [2]. Through this new service wholegenome sequencing (WGS) will become a routine and frontline test in cross-cutting areas of medicine [3].

The 100kGP framed participant consent as being of central importance (see Fig. 1 for 100kGP consent process). Indeed, one of its four main aims was 'to create an ethical and transparent programme based on consent' [4]. Consent 


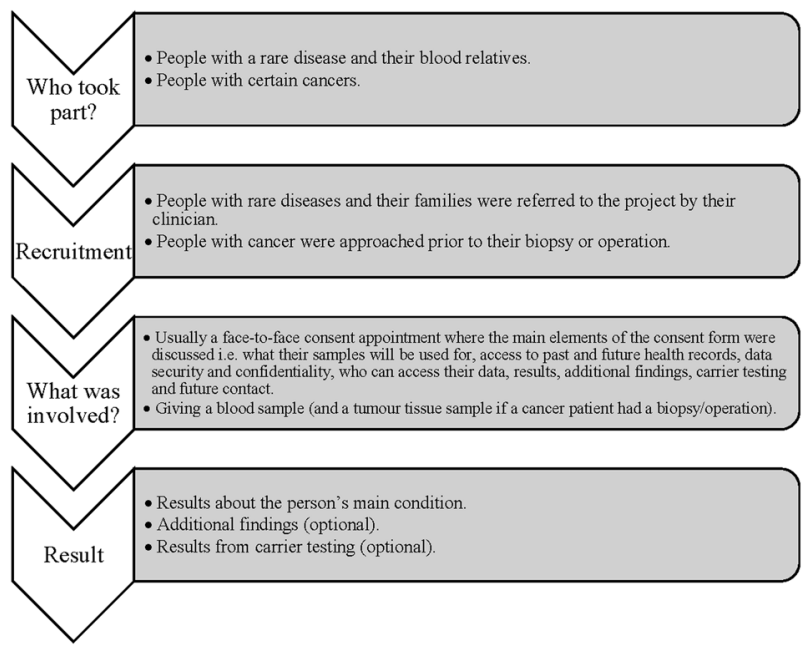

Fig. 1 Consent process in the 100,000 Genomes Project.

in the context of genomic testing has long been seen as a challenging issue and more so with the hybrid nature of the 100kGP, where research and clinical elements were combined in the consent approach. The detailed but unfocussed approach of genome sequencing, and its familial nature, means that results can be hard to accurately forecast and might be unexpected, or may have ramifications for others beyond the person being tested [5].

Research ethics committees tend to place great weight on the importance of consent, partly in reaction to previous scandals where research participants have been lied to or kept unaware of important information [6]. Clinical practice also elevates information provision during the consent process as the central method by which respect is shown for patient autonomy [7]. The many uncertainties that surround genomics [8] make specific consent hard to achieve, and correspondingly, 100kGP participants were at some points asked to make broad rather than specific decisions; for example, they could decide whether to receive AFs or not, but they could not pick and choose what these AFs might be [1].

We undertook a mixed method study: in that we used a survey to generate quantitative data regarding participants' recollections of the consent process for the 100kGP, and then explored the insights gained from the survey data more deeply with subsequent qualitative interviews that aimed to elicit how and whether consent for genomic testing appeared to be working in practice. This forms part of a wider study involving interviews with patients [9] and focus groups with health professionals (HPs) [10] regarding views on consent and confidentiality in relation to genetic information.

\section{Materials and methods}

This study used a survey followed by in-depth interviews with a subset of survey respondents [Fig. 2], aiming to

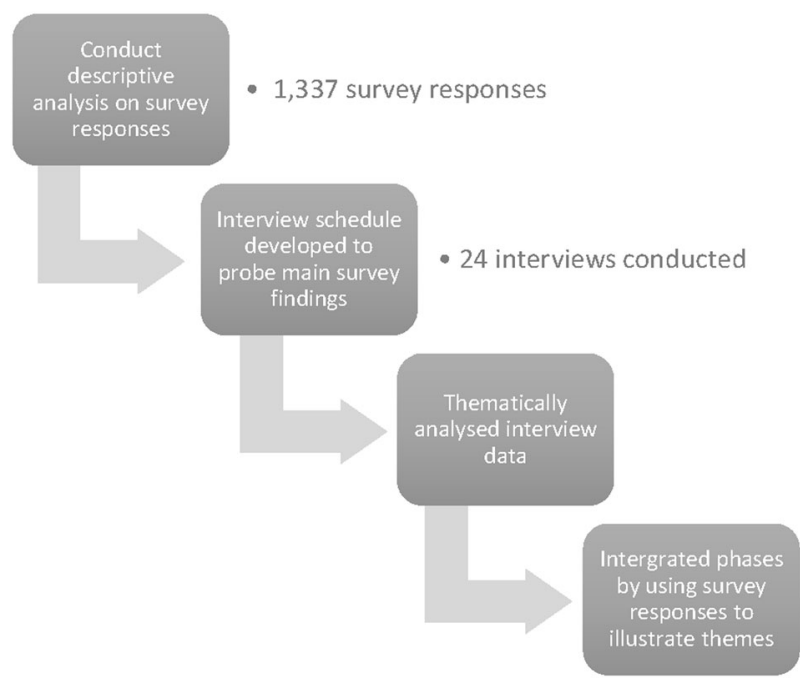

Fig. 2 A mixed method design.

further explore the findings of the survey and capitalise on the strengths of both qualitative and quantitative data in order to enhance the robustness of our conclusions [11]. Ethics approval was obtained from the NHS South Central Hampshire Research Ethics Committee (reference number 13/SC/0041).

\section{Development of survey and interview schedule}

We developed initial survey questions based on a review of the extant literature about ethical issues in genomics (see Supplementary Information for survey questions). Coauthors discussed each question and reached a consensus about which to keep. Discussion focused on whether the proposed questions were likely to elicit meaningful data about the consent approach in the context of the 100kGP [12]. In this paper we have focused on a selection of questions from the survey and reported interview data that linked to these questions, focusing on expectations regarding results, AFs and familial communication.

\section{Recruitment}

We recruited participants through one Genomic Medicine Centre (GMC), which comprised nine NHS trusts and served 3.5 million people. Participants were NHS patients with a rare disease, their families (often patient's parents, but sometimes other affected family members) and patients with cancer. HPs and research staff recruiting participants to the 100kGP handed out our survey to all participants aged 18 years or over who consented to take part in the 100kGP with an accompanying information sheet that explained the purpose of the research. Respondents either completed the survey at that time or completed it later and then returned it by post. In line with guidelines from the UK Health 
Research Authority [13], we inferred participant consent on receipt of a completed survey.

We have recruited 1819 participants to the survey study in total. However, early in the course of the study, we revised the survey, and in this paper we report on the revised survey only, which was completed by 1337 participants. Recruitment for the revised version of the survey took place from January 2017 to October 2018 with a response rate of $60 \%$ (the GMC recruited 3088 people to the $100 \mathrm{kGP}$ during this period, of whom $28 \%(n=865)$ were $<18$ and so not eligible to receive a survey). The survey contained an expression of interest slip regarding the interview study and participants could choose to fill this in to indicate their willingness to be interviewed. Forty-two percent $(n=562)$ of survey responders expressed an interest in being interviewed. Of interested survey responders a purposeful sample of $10 \%(n=54)$, which included participants from a range of conditions, ages and gender, were contacted by email or telephone. Of these, 24 were interviewed between May 2017 and April 2018 (24 did not respond to email or phone contact, and a suitable date could not be found for six). If they wished to proceed, a mutually convenient time and place was arranged. If participants came to the hospital, they were offered compensation for their travel and parking. All interviews were conducted by LB, who has a health psychology background and previous experience of interviewing people living with genetic conditions.

\section{Data analysis}

Interviews were transcribed and analysed thematically [14]. We generated codes from the first few transcripts and used these to guide the coding of all transcripts; codes were added to the analysis as subsequent transcripts were analysed. Codes were organised into categories and then refined into two overarching themes. We considered each of these themes in light of our survey data. NVIVO (QSR International, v11.4.3 (2084) for Mac) was used to organise and manage the qualitative data and SPSS (IMB Statistical Package for the Social Sciences v24) to conduct descriptive statistics for the survey data. No surveys were removed from the analysis if some data were missing so figures in results do not always total $100 \%$.

\section{Results}

\section{Demographics}

We surveyed and interviewed respondents from a range of ages, genders and education levels-Table 1 shows the participant demographics. Overall, over $70 \%$ of participants
Table 1 Demographics for survey and interview participants ${ }^{\mathrm{a}}$.

\begin{tabular}{|c|c|c|}
\hline Variable & Survey $(n(\%))$ & Interview $(n(\%))$ \\
\hline \multicolumn{3}{|l|}{$100 \mathrm{kGP}$ arm } \\
\hline Rare disease & $836(72.1)$ & $18(78)$ \\
\hline Cancer & $300(25.9)$ & $5(22)$ \\
\hline \multicolumn{3}{|l|}{ Respondent } \\
\hline Patient & $638(55)$ & $11(48)$ \\
\hline Patient's parent & $476(41)$ & $11(48)$ \\
\hline Other family member & $14(1.2)$ & $1(4)$ \\
\hline \multicolumn{3}{|l|}{ Gender } \\
\hline Woman & $640(55.2)$ & $16(70)$ \\
\hline Man & $487(42)$ & $7(30)$ \\
\hline Other & $7(0.6)$ & - \\
\hline \multicolumn{3}{|l|}{ Age } \\
\hline Under 20 & $18(1.6)$ & - \\
\hline $20-30$ & $79(6.8)$ & $2(9)$ \\
\hline $31-40$ & $232(20)$ & $6(26)$ \\
\hline $41-50$ & $213(18.4)$ & $7(30)$ \\
\hline $51-60$ & $183(15.8)$ & $8(35)$ \\
\hline $60+$ & $403(34.7)$ & \\
\hline \multicolumn{3}{|l|}{ Education } \\
\hline No schooling & $30(2.6)$ & 0 \\
\hline Primary & $50(4.3)$ & 0 \\
\hline GCSE or equivalent & $372(32.1)$ & 4 \\
\hline A level or equivalent & $249(21.5)$ & 5 \\
\hline Bachelor's & $215(18.5)$ & 11 \\
\hline Master's & $85(7.3)$ & 1 \\
\hline Doctorate & $21(1.8)$ & 0 \\
\hline Other & $70(6)$ & 0 \\
\hline
\end{tabular}

${ }^{\mathrm{a}}$ Figures do not always total 1337 due to missing data

came from the 'rare disease' arm of the 100kGP, reflecting the $74 \%$ of rare disease participants recruited by the GMC in total. A higher proportion of women were interviewed than men; we attempted to redress this but were unsuccessful (see Table 2 for a comprehensive account of the survey results).

The following expands on our two themes.

(1) 'I don't remember, maybe I didn't understand it completely".

This theme describes some participants' struggle to call to mind the nature of the decisions they had been asked to make; the various misconceptions they held about the project; and that many were unaware of key implications of the project, for example the potential relevance for family members, or the likelihood of finding a diagnosis.

Not all participants could recollect what decisions they had taken regarding whether to have AFs. This was more common in participants in the cancer arm of the project of whom $20 \%(n=67)$ were 'not sure' if they had consented to have AFs, relative to only $5 \%(n=49)$ of rare disease 
Table 2 Comprehensive survey results.

Did you decide to have additional findings?

Answer options Yes

Responses (\%) $\quad$ RD: 90

Cancer: 67 Cancer: 13 Cancer: 20

What will additional findings tell you?

$\begin{array}{llll}\text { Answer options } & \text { Serious risks } & \begin{array}{l}\text { Risks that are } \\ \text { not certain }\end{array} & \text { All kinds of possible risks } \\ \text { Responses }(\%) & \text { RD: } 3 & \text { RD: } 26 & \text { RD: } 71 \\ & \text { Cancer: } 4 & \text { Cancer: } 18 & \text { Cancer: } 78\end{array}$

Have you told your family members your genome is being tested?

Answer options $\quad$ Yes

$\begin{array}{lll}\text { RD: } 80 & \text { RD: } 12 & \text { RD: } 8\end{array}$

Cancer: 56 Cancer: $16 \quad$ Cancer: 28

Have you told family members about the search for additional findings?

Answer options Yes No

$\begin{array}{lll}\text { Responses (\%) } & \text { RD: } 63 & \text { RD: } 37\end{array}$

Cancer: $59 \quad$ Cancer: 41

How likely do you think it is that you'll get information about you/your family member's diagnosis?

\begin{tabular}{lllll} 
Answer options & Very likely & Likely & Unlikely & Very unlikely \\
Responses $(\%)$ & RD: 14 & RD: 48 & RD: 35 & RD: 3 \\
& Cancer: 19 & Cancer: 43 & Cancer: 30 & Cancer: 8 \\
\hline
\end{tabular}

$R D$ rare disease

participants. However, interview data suggested that poor recollection of decisions was perhaps more common than indicated by the survey as in some cases it became clear to the interviewer that a participant did not remember making a decision about AFs, despite indicating in the survey that they had chosen to find out about them (Table 3, quote 1). When these inconsistencies were pointed out to the participants, they appeared unconcerned. ${ }^{2}$

We asked participants about their recollections regarding the nature of AFs. Over two thirds of all survey participants (72\%, $n=755$ ) thought that AFs would tell them about 'all kinds of possible risks'. The 100kGP restricted AFs to a few select genomic variants known to predispose to serious conditions, for which treatment and/or screening is likely to be helpful. Less than $1 \%$ of participants are expected to have an $\mathrm{AF}$ under the current list $^{3}$ and the 100kGP participant information sheets stated that 'The diseases we look for are uncommon, and the chance of you having one of them is low' [15].

Of those interview participants who did remember providing consent for AFs, many had misunderstood what this information would tell them (Table 3, quote 2). Interview participants thought that AFs may tell them about their risk of developing conditions like arthritis, Huntington's, brain cancer

\footnotetext{
2 The researcher alerted the $100 \mathrm{kGP}$ team if a discrepancy was found and the team contacted the participant.

${ }^{3}$ As yet, no AFs have been reported to participants.
}

and Parkinson's, none of which are being searched for by the 100kGP. A few participants reported that they believed AFs would tell them about conditions that they already had, or those that would need 'immediate attention' (P9 rare disease).

The 100kGP participant information stated that the project might 'find something that could be important for the health of your family' [15]; and we asked questions about the familial implications of participating. Our survey found that over three-quarters of participants $(77 \%, n=997)$ reported that they had told their family members they were taking part in the project, whereas less than two thirds $(62 \%, n=737)$ had told those family members that AFs might be found as well as a main finding.

We explored this further during interviews, where some participants discussed how it had not occurred to them to inform their relatives (Table 3, quotes 3 and 4). Some did not understand why their results would be relevant to their relatives and thought AFs were personal to them: "I think the additional is probably more personal to me isn't it?" (P6 rare disease parent). Some interview participants explained that they intended to inform relatives but had not 'through lack of opportunity' (P17 rare disease parent) or plan to 'if something [an AF] comes out' (P14 cancer). Also, many indicated that the people that they chose to talk to about the project were not blood relatives for whom the project might find medically relevant information, but genetically unrelated family members whose support and opinion was important (Table 3, quote 5). 
Table 3 Quotes to illustrate the theme "I don't remember, maybe I didn't understand it completely".
1 I don't remember. I think I would have freely consented to that, because having gone into it, why not, you know. So, if I didn't then, I'll certainly do now. (P8 rare disease patient)

2 I don't remember what the additional findings were to be honest, but I agreed to it. (P7 cancer)

3 I possibly hadn't even thought of the wider blood relatives. Just kind of initially you think of yourself. (P11 rare disease patient)

$4 \quad$ Maybe I should have [told relatives about participating in the 100kGP], but then if someone had said that to me, go home and speak to your family about it, then I would have thought "oh yeah actually", but it's only you speaking about it now that I actually stop to think about them. (P1 rare disease patient)

5 Interviewer: Did you talk to them [family] about the additional findings as well?

P5 cancer: Yeah, I did, and my husband was, he said, that'd be a good thing [having AFs looked for], because obviously knowledge and prevention is better than waiting for it to happen, isn't it?

Interviewer: And did you have a discussion with people that are related to you? Whatever is found for you won't be relevant to your husband.

P5 cancer: No.
We asked questions about the likelihood that they or their family members would receive a diagnosis through the 100kGP. Participants tended to hold optimistic views about what they would get from the project. Over half of survey participants $(62 \%, n=693)$ thought that it was likely $(48 \%$, $n=533)$ or very likely $(14 \%, n=160)$ that they, or their family member, would receive a diagnosis. In contrast, the 100kGP report on their website that an estimated 20-25\% of participants will receive a diagnosis [15], though this was not included in the information sheets or consent forms.

(2) "I don't remember much, and I don't understand everything, but that's OK".

This theme describes how many participants seemed unconcerned that they could not recollect some details of what they consented to- they trusted that HPs, and the project, would act in their interests.

As survey data showed that some participants did not recall the decisions they had made and had not understood certain aspects of the project, we explored this with interview participants. Participants were aware that they could not remember everything; they may have remembered certain aspects, but rarely the details. What they did report was that they felt satisfied with the consent process and had been given enough information to make a decision about whether or not to participate and have AFs looked for (Table 4, quote 1).

Interviewees did not think the project was particularly complicated. When asked about the consent process, and if anything could have been made clearer, many said the project was clearly explained, made sense and was 'straightforward'. Trust appeared to play a part in why participants took the decision to participate, a finding also reported in other studies [16-20]. Participants were not worried about the technicalities and trusted that the researchers would use their data responsibly (Table 4 , quote 2 ), with the number of documents they received enhancing the perception of thoroughness and reliability (Table 4, quote 3 ). We specifically asked interview participants if they had concerns about their data being held electronically and all but one indicated they had no worries. The participant who did have concerns had made the decision to participate regardless as they felt that the potential benefits of participating outweighed these concerns (Table 4, quote 4). Participants put aside any concerns they had and put themselves in the hands of the expert: "You have to trust these people. They've spent years in training $[. .$.$] you have to put it$ [trust] into the HPs" (P10 cancer), with some participants feeling happy to sign the consent form before their HP felt comfortable to let them do so (Table 4, quote 5). The view that the project was trustworthy appeared to stem from several sources, for example participants attributed certain qualities to the project, such as not revealing information to insurance companies (Table 4, quote 6), written information about the project (Table 4, quote 7), investment in adjunct social research (Table 4, quote 8), positive past experience of the NHS (Table 4, quote 9) and specific mechanisms to preserve confidentiality (Table 4, quote 10).

Some participants were aware that they might be contacted in the future-after they had received their 'main result', since researchers would continue to look at their data and new evidence might emerge. These participants felt more relaxed about not being able to recall decisions, or understand exactly what results they would get, because they assumed this would be revisited in the future if necessary (Table 4, quote 11). Other participants assumed that an initial result letter was all that they would receive, and thought, wrongly, that their letter relating to 'main findings' meant that AFs had been checked for too (Table 4, quote 12).

\section{Discussion}

In this mixed method study, we found that many participants in the 100kGP did not always remember the decisions they were asked to make during the consent process. They 
Table 4 Quotes to illustrate the theme "I don't remember much, and I don't understand everything, but that's OK".

1 I did read all of the stuff at the time, and I can't really remember what it says, but I'm not too concerned [...] everything that I've read about what you guys are doing, there isn't anything that really worries me. (P6 rare disease parent)

2 I presume it'll [data] be put in, in trials or something. I don't know. I mean I know it's [...] anonymised and everything [...] I'll leave it to the researchers to use as they want. I'll leave it to them. (P3 rare disease patient)

3 I think it's all really quite straightforward and explained very well, so quite a lot of documents; you know literature that's come with it, so it's good." (P6 rare disease parent)

$4 \quad$ Such brilliant stuff is going to come from it, that you just suck it up almost. (P13 rare disease parent)

5 I was a bit like, it doesn't matter what you want me to do, I'll do it anyway, but she was very much like, well let's just talk about it, and let's take time, and she did spend time with me going through it. And at the time, I was a bit, oh you don't have to, it's fine, I'll just sign it anyway, and she was no, I want to talk to you about it and make sure you understand it. So, yeah, it seemed fairly straightforward. (P12 cancer)

6 They're not going to be ringing up my insurance companies and things like that. (P03 rare disease patient)

7 Everything that I've read about what you guys are doing, there isn't anything that really worries me. (P06 rare disease parent)

$8 \quad$ You've had so many surveys come through and you've probably spoken to numerous different people, so you get a feeling and I think you've got to trust that. (P10 rare disease parent)

9 The NHS have done nothing but brilliant stuff for us. (P12 cancer)

10 They kept saying that his, he'll just be a number, he won't be, nobody will be able to identify him, not that I'm worried. (P19 rare disease parent)

11 I think they talked about something that it can be kept for a while and then used again for something else, if something happens later on and there are new developments, they might go back to it. (P12 cancer)

Most people would think they've got a letter saying they didn't find anything in me. And they didn't find I was a carrier, so I naturally presumed that they'd looked into everything, I'm fine. (P24 rare disease patient)

also had various misconceptions about what sort of results they might receive from the project and, in some cases, were unaware that the project might find health information relevant for their wider family. Participants tended to have an optimistic view of the likelihood of finding a diagnosis via the 100kGP, and most felt satisfied with their decision to participate, even when they were made aware that the decisions they appeared to take during the consent process were different to what they thought. Our study demonstrates that many participants do not appear to have given consent to take part in the 100kGP based on scrutinising and weighing up the large volumes of information provided by the 100kGP, but instead because they trusted the HP that suggested that they consider taking part, and trusted the project itself [20]. However, some participants may have been strongly weighted towards participating to be able access technological advances they (or their children) would not otherwise be offered. It is possible they felt 'coerced by circumstance' and had to put aside any concerns they may have; as one participant explained "If you had told me that you were going to sell my information to the Russians, then I probably would have still done it" (P6 rare disease parent).

\section{Facilitating decision-making during consent conversations}

Our study has important implications for future practice regarding how patients' consent is sought for genomic testing-especially considering the complexity hybrid clinical-research endeavours introduce-and what weight is subsequently attributed to the decisions taken during an initial consent conversation. The 100kGP approach, with its strong emphasis on comprehensive written information and lengthy consent consultations, clearly engendered trust in participants and was viewed positively, but perhaps because of functions other than information provision. The number of documents participants received may have enhanced the perception of thoroughness and reliability, acting like 'symbolic tokens' (17, pg 2220) of legitimacy and trustworthiness. Whilst recent court cases have tended to focus on the adequacy of information provision (e.g. Montgomery ${ }^{4}$ ), provision of information is only part of the consent process. Dickert et al. argue that consent is richer than respecting patient autonomy, recall of information and signing a form [21]. Our study supports this, finding that the consent approach in the 100kGP encompassed additional ethically important functions, such as reinforcing trust.

Our data suggest that if the consent process for complex ongoing investigations-such as genomic testing-is judged solely on participants' ability to accurately recall the decisions they took, it would need rethinking. For example,

\footnotetext{
${ }^{4}$ The Montgomery ruling (2015) established that it is not for a medical professional to decide what information to provide to a patient. Instead health professionals need to provide information that a reasonable patient would want to know as well as what the particular patient in question wants to know. The medical professional is or should reasonably be aware that the particular patient would be likely to attach significance to a risk of injury in treatment.
} 
some participants could not remember, or incorrectly remembered, whether they had asked for AFs to be looked for. This is in keeping with a previous interview study with rare disease participants from a genome sequencing project [22], where the study team demonstrated that interviewees who thought they had declined AFs - and stated their reasoning behind this decision-had actually consented to receive AFs during the consent process. Moreover, $61 \%$ of our survey participants thought that AFs would tell them about 'all kinds of possible risks', rather than a narrow menu of serious conditions for which screening and/or treatment is likely to be available. Whilst thinking AFs might be broader than they are is not necessarily harmful, it is concerning that some interviewees-who expressed that they had not chosen to find out about AFs-had ostensibly chosen to do so when they provided consent for the project (and vice versa). Rigidly sticking to patients' binary answers to complex questions made some time ago, when there is little evidence that these answers reflect what they think today, may prove to be ill-advised [23].

This in turn presents a challenge to the usefulness of consent forms; what patients thought they had chosen, and what they had indicated at the time of consent, were at times different. This suggests that when difficult ethical questions arise in the clinic, for example if a health risk is inadvertently found during genomic testing where a patient could mitigate the risk if they knew about it, we should not exclusively decide what to do by deferring to their previous consent forms. The consent process should be seen as a continuum of ongoing communication to allow for changes over time [24], and whilst the consent form might be a useful proxy for what a patient might currently think, our data suggest that this should not be assumed without question. It can be argued that in as much as a participant understands the decisions that they are making at the time of a consent conversation, their consent is 'informed', regardless of whether they can subsequently recollect the decisions they made. However, our research shows that people who have made decisions during the consent process would not necessarily endorse the decision that they took at that time when asked again at a later date. Whilst this does not necessarily mean that their original decision was not 'informed', it does mean that HPs should be aware that there is potentially a temporal aspect to 'informed consent', and a decision taken some time ago may not accurately reflect the decision that a participant might take today.

Our study also raises the question of how consent conversations functioned during the $100 \mathrm{kGP}$ - why did some participants apparently make different decisions to what they thought they had? Some people might have changed their mind over the months since joining the project, or perhaps had not engaged with questions in the same way when they were raised during their 100kGP consent conversation, as when they explored them during their indepth interview for this study. The $100 \mathrm{kGP}$ consent process packed in a large quantity of information [4], and our data suggest that on the whole this was not seen as problematic by patients. There is a tension between providing sufficient information such that people can make informed decisions about genomic testing, and providing so much information that they cannot meaningfully engage with some of these decisions [3].

Our research indicates a potential discrepancy between the choices participants might have documented on their consent forms for $100 \mathrm{kGP}$ regarding AFs, and the choices that they actually intended to make. Perhaps discussions about AFs needed more prominence during consent conversations, potentially at the expense of detailed discussion of issues like data security [25]. Whilst it is clearly very important that participants have access to detailed information about the latter, if they find it relevant and useful, we feel it is important to consider how to provide information on these topics without overshadowing discussion of other issues that patients might consider more important. In complex situations like these, whilst the decisions needing discussion may be broad in scope, consent discussions may need to be tailored in the sense that they need to focus on the aspects of greatest concern to the particular individuals making these decisions. This will mean HPs moving away from aiming to cover everything in a tick box-type model.

\section{Informing patients about genomic tests}

Some participants had not considered that their results might be relevant to their blood relatives, and many participants had unrealistic expectations of the likelihood of receiving a diagnosis or AF. The majority of survey participants thought it was likely that they or their family member would receive a diagnosis from the project, whilst the actual figures are likely to be much lower. These results are supported by findings from a survey of rare disease $100 \mathrm{kGP}$ participants conducted by Genetics Alliance and Genomics England who found a mismatch between participants' hopes of taking part and what has actually been delivered so far by the project [2]. Media discourse around genomics and personalised medicine - which tends to present the usefulness of genomic technology in a strongly positive light-may have contributed to creating high expectations as to what the $100 \mathrm{kGP}$ was able to deliver [26]. Our research emphasises the importance of highlighting the potential limitations of genomic testing during the consent process-many people will not receive a genomic diagnosis, or their results may be unclear and difficult to interpret [5].

The benefits of genomic testing, especially testing for pre-symptomatic treatable diseases, will be realised partly by patients sharing this information with their relatives [27]. 
Our findings reiterate the importance of ensuring that people having genomic testing are made aware that their decisions, and their results, may have relevance for their blood relatives. One participant expressed this particularly clearly: "If someone had said that to me, go home and speak to your family about it, then I would have thought "oh yeah actually", but it's only you speaking about it now that I actually stop to think about them". It appears paradoxical that the rare disease participants were taking part in a project with their family members but did not fully recognise that results from the project might have relevance for others in their family. Participants seemed to have compartmentalised certain findings, maybe this helped them understand this complex project. Our survey confirmed that the majority of participants had not told their relatives about their decision about AFs. Whilst this does not necessarily mean that they would not go on to inform their relatives if an AF was found, earlier awareness that genomic testing could reveal information of familial relevance might make this process easier [28]. Previous research indicates that patients generally recognise the importance of sharing genetic information with family members, especially regarding risks of diseases that can be prevented or treated [9], although, in practice, some patients struggle to inform their at-risk relatives in a timely fashion [29]. We suggest that during the consent conversations for genome sequencing, patients should be encouraged to consider talking to their relatives about their decision to have a test.

\section{Implications for the NHS Genomic Medicine Service}

Despite the inaccurate recollection and misperceptions about the project, participants generally felt satisfied with their decision to take part in the $100 \mathrm{kGP}$. Many participants expressed trust in the project and the HPs involved, and were unconcerned even when it was pointed out that some of the decisions they made during the consent process were different to what they had previously thought. This finding may be connected to trust that the project would 'do the right thing' regardless. If this is the case, then the project has a responsibility to continue acting in a trustworthy manner, which may involve adapting the existing consent process to include determining whether participants who consented to have AFs looked for are still happy to receive them (and vice versa).

Our study suggests that whilst consent conversations for the $100 \mathrm{kGP}$ did not always succeed at informing participants and eliciting what they really thought about particular questions, they were fulfilling wider functions such as reinforcing trust [21]. Some $100 \mathrm{kGP}$ participants will have chosen to take part based on trust rather than on carefully weighing and considering large volumes of information [20]. This underlines the importance of the newly formed
NHS Genomic Medicine Service focussing on trustworthiness by reflecting on empirical findings, from studies such as ours, and continuing to refine and research the consent process [30]. This trust needs to be maintained by ensuring that genomic testing takes place within a system of processes, where patients can be confident that their data will be protected appropriately, and that their preferences will guide the sorts of results that might be looked for [3, 31]. Part of this process might involve ensuring that patients are not given the illusion of clear-cut choices if these might later be hard to interpret and honour.

We argue that in the context of the NHS Genomic Medicine Service, consent conversations need to be more open-ended [32], with participants aware that aspects of their consent might need to be revisited over time in response to changing contexts. Findings from other studies support this, suggesting that patients would like more information and more contact throughout the process of genomic testing [2]. The consent and confidentiality guidelines in genomic medicine move towards this, offering a 'record of discussions' template as opposed to a consent form [33]. As genomic testing transitions from being available only via projects like the $100 \mathrm{kGP}$, with dedicated research time and infrastructure to support it, to being routinely offered in the NHS [31], we highlight the need to examine our practices regarding consent. This is reiterated by the Nuffield Council on Bioethics who outline the limitations of one-off consent in fast changing areas such as genomics, where outcomes are sometimes unexpected [30]. Nevertheless, genetics services are still using consent forms despite a record of discussions template being recommended in a previous edition of the consent and confidentiality guidelines [34]. Consent may not be operating in the ways that we expect, and further research is needed to explore strategies to improve patients' engagement with, and recollection of, the key decisions they are asked to make during consent conversations about genomic testing. We plan to further explore 100kGP participant experiences of receiving their main results and AFs.

\section{Strengths and limitations}

The response rate for our survey was $60 \%$ and we recruited participants from a broad range of ages and disease types. However, $15 \%$ of surveys returned had missing data. Studies reporting the experience of participating in the $100 \mathrm{kGP}$ often focus on participants with rare disease [2, 20, 25], whereas we have also explored the experience of participants with cancer. The results presented in this study are from one GMC, so we cannot say with certainty that these findings are representative of other GMC participant experiences. However, the consent documents and training for conducting consent appointments were standardised 
nationally. Some of the wording in the survey could have been interpreted differently by different people (e.g. what do 'likely' and 'information about a diagnosis' mean?). Due to the mixed methodology we were able to explore the findings from the survey in more detail in the interviews, to clarify points further and to explore wider topics.

\section{Conclusion}

Seeking participant views about the 100kGP is essential for ensuring that the NHS Genomic Medicine Service evolves in an ethically sound way, that is in a way that benefits and respects participants and their relatives as well as protecting them from potential harm. It is not surprising that a project such as this, with such diverse aims and participant groups, and blend of research and clinical aims and governance, would throw up at least some challenges. Our findings suggest that consent alone cannot bear the weight of all subsequent decisions about what findings to disclose from WGS. Consent was of central importance to the 100kGP; however, different aims were achieved through the consent process than were originally planned. Our research shows that some participants did not remember key details of decisions taken during their initial consent conversation and had expectations that differed from those the project could deliver, emphasising that genomic testing needs to happen in a context whereby these issues can be dealt with along the way. Providing participants with a copy of their consent forms, as the 100kGP did, may be a useful step in allowing people to remind themselves of the decisions they made at the time of their initial consent conversation. However, such an approach is not sufficient to conclude that a person still holds the same views now that they appeared to at the time of the consent conversation. We highlight the need for a national discussion about the role of consent in the NHS Genomic Medicine Service-how can we best facilitate it, and how should we respond to questions that patient consent alone cannot answer? Our paper raises the question: are participants in the 100kGP prepared for the issues that arise from not remembering or understanding discussions had and decisions made in the initial consent appointments?

\footnotetext{
Acknowledgements We wish to thank the staff recruiting participants to the 100kGP who handed out our survey, the patients who completed the survey and interview and Lisa Scott for her invaluable administrative support. LB is funded by a Research Fellowship from Health Education England Genomics Education Programme. RH is funded by a Wellcome Trust Research Award for Health Professionals (218092/ $\mathrm{Z} / 1 \mathrm{~g} / \mathrm{Z}$ ). This work was supported by funding from a Wellcome Trust collaborative award [grant number 208053/Z/17/Z (to AL)] and funding from the National Institute for Health Research (NIHR) Wessex Clinical Research Network. The views expressed in this publication are those of the author(s) and not necessarily those of the HEE GEP, NIHR or the Department of Health and Social Care.
}

\section{Compliance with ethical standards}

Conflict of interest The authors declare that they have no conflict of interest.

Publisher's note Springer Nature remains neutral with regard to jurisdictional claims in published maps and institutional affiliations.

\section{References}

1. Caulfield M, Davies J, Dennys M, Elbahy L, Fowler T, Hill S, et al. The 100,000 Genomes Project Protocol. 2017. Available from: https://www.genomicsengland.co.uk/wp-content/uploads/ 2017/03/GenomicEnglandProtocol_151117-v4-Wales.pdf.

2. Spencer-Tanslet R, Hunter A. Genome sequencing and the NHS. The views of rare disease patients and carers. London: Genomics England; 2019.

3. Davies SC. Annual report of the chief medical officer 2016. Generation genome. London: Department of Health; 2017. Available from: https://assets.publishing.service.gov.uk/ government/uploads/system/uploads/attachment_data/file/631043/ CMO_annual_report_generation_genome.pdf.

4. Samuel GN, Farsides B. Public trust and 'ethics review' as a commodity: the case of Genomics England Limited and the UK's 100,000 genomes project. Med, Health Care, Philos. 2018;21:159-68.

5. Horton R, Lucassen A. Consent and autonomy in the genomics era. Curr Genet Med Rep. 2019;7:85-91.

6. Beauchamp TL. The idea of a "standard view" of informed consent. Am J Bioeth. 2017;17:1-2.

7. General Medical Council. Consent: patients and doctors making decisions together. London: General Medical Council; 2008.

8. Howard HC, Iwarsson E. Mapping uncertainty in genomics. J Risk Res. 2017;21:117-28.

9. Dheensa S, Fenwick A, Lucassen A. 'Is this knowledge mine and nobody else's? I don't feel that.' Patient views about consent, confidentiality and information-sharing in genetic medicine. $\mathrm{J}$ Med Ethics. 2016:42:174-9.

10. Dheensa S, Fenwick A, Lucassen A. Approaching confidentiality at a familial level in genomic medicine: a focus group study with healthcare professionals. BMJ Open. 2017;7:e12443.

11. Ivankova NV, Cresswell JW, Stick SL. Using mixed-methods sequential explanatory design: from theory to practice. Field Methods. 2006;18:3-20.

12. Hirai K, Shiozaki M, Motooka H, Arai H, Koyama A, Inui H, et al. Discrimination between worry and anxiety among cancer patients: development of a brief cancer-related worry inventory. Psychooncology. 2008;17:1172-9.

13. NHS Health Research Authority. Informing participants and seeking consent. NHS Health Research Authority. 2019. https://www.hra.nhs.uk/planning-and-improving-research/best-pra ctice/informing-participants-and-seeking-consent/.

14. Braun V, Clarke V. Using thematic analysis in psychology. Qual Res Psychol. 2006;3:77-101.

15. Genomics England. Information for participants. Genomics England. 2018. https://www.genomicsengland.co.uk/taking-part/ information-for-participants/.

16. Armstrong N, Dixon-Woods M, Thomas A, Rusk G, Tarrant C. Do informed consent documents for cancer trials do what they should? A study of manifest and latent functions. Sociol Health Illn. 2012;34:1230-45.

17. Dixon-Woods M, Ashcroft RE, Jackson CJ, Tobin MD, Kivits J, Burton PR, et al. Beyond "misunderstanding": written information 
and decisions about taking part in a genetic epidemiology study. Soc Sci Med. 2007;65:2212-22.

18. Kenyon S, Dixon-Woods M, Jackson CJ, Windridge K, Pitchforth E. Participating in a trial in a critical situation: a qualitative study in pregnancy. Qual Saf Health Care. 2006;15:98-101.

19. Dixon-Woods M, Tarrant C, Jackson C, JONES D, Kenyon S. Providing the results of research to participants: a mixed-method study of the benefits and challenges of a consultative approach. Clin Trials. 2011;8:330-41.

20. Dheensa S, Lucassen A, Fenwick A. Fostering trust in healthcare: participants' experiences, views, and concerns about the 100,000 genomes project. Eur J Med Genet. 2019;62:335-41.

21. Dickert NW, Eyal N, Goldkind SF, Grady C, Joffe S, Lo B, et al. Reframing consent for clinical research: a function-based approach. Am J Bioeth. 2017;17:3-11.

22. Mackley MP, Blair E, Parker M, Taylor JC, Watkins H, Ormondroyd E. Views of rare disease participants in a UK wholegenome sequencing study towards secondary findings: a qualitative study. Eur J Hum Genet. 2018;26:652-9.

23. Horton R, Bell B, Fenwick A, Lucassen AM. Is it acceptable to contact an anonymous egg donor to facilitate diagnostic genetic testing for the donor-conceived child? J Med Ethics. 2019;45:357-60.

24. Laurie G, Postan E. Rhetoric or reality: what is the legal status of the consent form in health-related research? Med Law Rev. 2013;21:371-414.

25. Sanderson SC, Lewis C, Patch C, Hill M, Bitner-Glindzicz M, Chitty LS. Opening the "black box" of informed consent appointments for genome sequencing: a multisite observational study. Genet Med. 2019;21:1083-91.

26. Marcon AR, Bieber M, Caulfield T. Representing a "revolution": how the popular press has portrayed personalized medicine. Genet Med. 2018;20:950-6.
27. Tibben A, Biesecker BB. Clinical genome sequencing: psychological considerations. London: Elsevier; 2019.

28. Eijzenga W, de Geus E, Aalfs CM, Menko FH, Sijmons RH, de Haes $\mathrm{H}$, et al. How to support cancer genetics counselees in informing at-risk relatives? Lessons from a randomized controlled trial. Patient Educ Counsel. 2018;101:1611-9.

29. Chivers Seymour K, Addington-Hall J, Lucassen AM, Foster CL. What facilitates or impedes family communication following genetic testing for cancer risk? A systematic review and metasynthesis of primary qualitative research. J Genet Counsel. 2010;19:330-42.

30. Nuffield Council on Bioethics. The collection, linking and use of data in biomedical research and health care: ethical issues. Nuffield Council on Bioethics. 2015. Available from https:// nuffieldbioethics.org/publications/biological-and-health-data.

31. Dheensa S, Samuel G, Lucassen AM, Farsides B. Towards a national genomics medicine service: the challenges facing clinical-research hybrid practices and the case of the 100000 genomes project. J Med Ethics. 2018;44:397-403.

32. Department of Health. Reference guide to consent for examination or treatment. 2nd ed. Department of Health. 2009. Available from https://www.gov.uk/government/publications/reference-guide-toconsent-for-examination-or-treatment-second-edition.

33. Lucassen A, Hall A. Consent and confidentiality in genomic medicine. 3rd ed. Royal College of Physicians. 2019. Available from https://www.rcplondon.ac.uk/projects/outputs/consent-andconfidentiality-genomic-medicine.

34. Dheensa S, Crawford G, Salter C, Parker M, Fenwick A, Lucassen A. How do clinical genetics consent forms address the familial approach to confidentiality and incidental findings? A mixed-methods study. Fam Cancer. 2018;17: $155-66$. 